2021, Band 9, Heft 1/2

Seiten 165-170

zeitschrift-suburban.de

10.36900/suburban.v9i1/2.674

\title{
The end of care-less capitalism (as we knew it)?
}

Kommentar zu Stefan Höhne und Boris Michel „Das Ende des Städtischen?

Pandemie, Digitalisierung und planetarische Enturbanisierung“

Stefan Höhne, Boris Michel ,Das Ende des Städtischen? Pandemie, Digitalisierung und planetarische Enturbanisierung'

Kommentare von:

Marcelo Lopes de Souza, Matthias Naumann, Anke Strüver, Markus Kip, Hannah Schilling, Roger Keil, Christian Haid, Anna-Lisa Müller, Nikolai Roskamm

\section{Anke Strüver}

\section{Pandemischer Urbanismus}

„Das Ende des Städtischen?“ fragen Stefan Höhne und Boris Michel (2021) in ihrem Debattenaufschlag und tragen viele Beobachtungen zusammen, die dafür - und dagegen - sprechen. Mich haben diese Frage sowie einige der aufgelisteten Symptome direkt an J. K. Gibson-Grahams (2006) „The end of capitalism (as we knew it)" und an die Rolle der Sorgearbeiten im Pandemischen Urbanismus erinnert. Denn in den sich mittlerweile häufenden harten Lockdowns erfolgt die Sicherung der Grundbedürfnisse durch sorgende Tätigkeiten. Als ,systemrelevant" werden (wenn auch unzureichend) jene Versorgungs- und Sorge-Dienstleistungen anerkannt, die die Grundbedürfnisse durch Arbeit im Supermarkt, im Krankenhaus und im Bereich der Pflege sichern. Was aber ist mit den Sorgearbeiten zu Hause? In Zeiten des angeordneten Rückzugs ins Private, geschlossener Schulen, Kitas, Bars und Restaurants sowie der Einrichtung provisorischer Homeoffices führen die Lockdowns zu einer Vervielfachung der Aufgaben und zur Verdichtung der Körper zu Hause. Insbesondere für Eltern kommt durch Homeschooling und Freizeitgestaltung neben dem Einkaufen, Kochen und Abwaschen eine zeitlich wie räumlich intensivere Nutzung des Wohnraums hinzu, die zu vermehrten Aufräum- und Putzarbeiten führt. In Mitteleuropa - auf das ich mich hier vorrangig beziehe - nehmen viele Menschen erst während der Lockdowns wahr, was alles Sorgearbeiten sind und was alles in den letzten Jahrzehnten an Sorgearbeiten extern erledigt worden ist - außer Haus, wie Kinderbetreuung, Pflege und Verpflegung, oder zu Hause, aber durch entlohnte Dienstleister_innen.

Die Pandemie hat uns die Auswirkungen der Globalisierung vorgeführt. Die Coronakrise hat uns zugleich die Unumgehbarkeit lokaler, alltäglicher physischer wie psychischer Sorgearbeiten - entlohnter wie unentlohnter - spüren lassen. Sie intensiviert die Carekrise[1] und die kapitalistische Krise und muss daher auch in diesen Zusammenhang gestellt werden. Die folgenden Abschnitte nehmen diesen Zusammenhang auf und diskutieren kurz die Reorganisation von Reproduktions- und Sorgearbeit 
mit Blick auf die feminisierte unsichtbare Arbeit im privaten Wohnraum (2), die potenziellen Veränderungen, die sich durch plattformvermittelte Sorgedienstleistungen ergeben (3), und ausblickend Überlegungen für eine Sorgende Urbanisierung (4).

\section{Planetarische Urbanisierung}

In den Thesen zur Planetarischen Urbanisierung - wie auch zum „Recht auf Stadt" - spielt das Sorgen kaum eine Rolle und das Versorgen ist reduziert auf die Diskussion um Tausch- und Gebrauchswert. Nina Schuster und Stefan Höhne (2017) haben in ihrer Einführung zum Themenschwerpunkt „Stadt der Reproduktion“ die Unsichtbarkeit von Sorge- und Reproduktionsarbeit[2] thematisiert - eine Unsichtbarkeit, die sich aus dem gesellschaftlichen Alltag auch in die Forschung überträgt. Während der Lockdowns werden Sorgeaufgaben jedoch so überlebensnotwendig und selbstverständlich, dass die Verbindung von Sorgearbeit und Stadt sicht- und spürbar sowie als Zusammenhang von Coronakrise und Carekrise zu adressieren ist.

Die feministische Raumforschung erklärt diese Unsichtbarkeit mit dem Ort und der Vergeschlechtlichung von Sorgearbeit: Sie ist traditionell wie aktuell vorrangig weiblich konnotiert und wird mit dem privaten Wohnraum assoziiert: Sie basiert auf der vergeschlechtlichten und verräumlichten Arbeitsteilung des Fordismus (außerhäusliche Lohnarbeit und häusliche Reproduktionsarbeit) und hat sich im städtebaulichen Ideal der räumlichen Funktionstrennung manifestiert. Durch den wachsenden Anteil von Frauen, die einer bezahlten außerhäuslichen Tätigkeit nachgehen, hat sich diese Arbeitsteilung jedoch verändert: Viele Sorgeaufgaben wurden vermarktlicht und im Zuge dessen personell wie räumlich ausgelagert (Kinderbetreuung, Verpflegung, Reinigung etc.). Das heißt, während die Debatte der 1970er Jahre die Rolle unbezahlter Hausarbeit für die Reproduktion der entlohnten Arbeitskräfte und das Funktionieren des Kapitalismus betont hatte, muss für die neoliberalen Umstrukturierungen geschaut werden, wie Sorgearbeit vermarktlicht, enthäuslicht und entlohnt wird. Dabei muss erstens das Verhältnis zwischen bezahlter und unbezahlter Sorgearbeit thematisiert und zweitens berücksichtigt werden, dass bezahlte Sorgearbeit eine prekarisierte ist, da sie für Profit- mittels Produktivitätssteigerungen ungeeignet ist (Federici 2019; Soiland 2020).[3]

Anders als die Diskussionen zur Privatisierung von urbanen Infrastrukturen gehört die Vermarktlichung der Sorgearbeit zur Reprivatisierung im engeren Sinne - und während der Lockdowns wird diese Reprivatisierung durch das Verbot von Öffentlichkeit angeordnet praktiziert: Sorgearbeiten im Privatraum haben sich vervielfältigt und verdichtet - und haben aufgrund von inkorporierten Rollen und Erwartungen zu einer Retraditionalisierung der vergeschlechtlichten Arbeitsteilung in heteronormativen Familienkonstellationen geführt.

Bereits vor der Pandemie und den sich in kürzester Zeit verändernden Wohn- und Arbeitsformen war klar, dass Lohnarbeit im Homeoffice als,Lösung der Carekrise erstens nur für Beschäftige der Wissensökonomie relevant sein kann und dass diese Klassenfrage zweitens auch eine der Geschlechterverhältnisse ist: Die Grundidee der Telearbeit, also eines raum- und zeitunabhängigen 
Arbeitens am Computer, war die einer besseren Vereinbarkeit von Beruf und Familie. Sie hat jedoch nicht zu einer Umverteilung von unentlohnten Sorgeaufgaben zu Hause geführt, sondern insbesondere für Mütter die individuelle Hausfrauisierung intensiviert (Carstensen 2020).

Während der Lockdowns haben zum einen das Arbeiten im Homeoffice und zum anderen Sorgearbeiten das angeordnete Abstandhalten und $\mathrm{Zu}$ hausebleiben überhaupt erst ermöglicht. Nur unzureichend diskutiert wird aber, dass keine_r eine Ganztagsversorgung von Kindern (Schule, Freizeit, Verpflegung etc.) und Lohnarbeit im Homeoffice zur gleichen Zeit und am gleichen Ort bewerkstelligen kann. Noch im Coronajahr 2020 gab es zahlreiche Studien, die sich mit der Verlagerung der Homeofficearbeit von Frauen in die Nachtstunden, in den Keller usw. beschäftigt haben (Kohlrausch/ Zucco 2020). Diese widerlegen auch die zu Beginn der Pandemie angestellten Vermutungen, dass Homeofficearbeit von Vätern zu mehr Geschlechtergerechtigkeit führe, da sie im Lockdown mehr Zeit zu Hause verbringen (Craig 2020; Derndorfer et al. 2020).

\section{Plattformurbanismus}

Wie oben erwähnt ist entlohnte Sorgearbeit für Profitmaximierung uninteressant. Sie wird gleichwohl zunehmend über Plattformen vermittelt, die nicht an der Sorgearbeit verdienen, sondern an ihrer Vermittlung - und an der Weiterverarbeitung der dabei generierten Daten.

Parallel zum Herunterfahren der Wirtschaft haben durch die Lockdowns einige der urbanen Plattformökonomien an Bedeutung gewonnen, die sich zur ökonomisierten Antwort auf die neoliberale Carekrise entwickelt haben. Insbesondere Plattformen für Essenslieferungen steigerten ihren Umsatz in den Städten des globalen Nordens: doordash.com beispielsweise meldete für die ersten fünf Monate der Pandemie ein Nachfrageplus von 110 Prozent in den USA und erklärt dies mit ihrem Ansatz, anders als die Konkurrenz, auch in die Vororte - nicht nur die Kernstädte - zu liefern. Wie sich aus Bestellzeiten und -zusammensetzung erschließen lässt, wurde nun verstärkt auch an Menschen im Homeoffice sowie an Familien geliefert.[4] Auf Plattformen für Pflege-, Betreuungs- und Reinigungstätigkeiten hingegen sank aufgrund des Kontaktverbots die Vermittlungsaktivität; für die Niederlande haben van Doorn/Mos/Bosma (2020) einen Umsatzrückgang bei helpling.com auf 40 Prozent festgestellt.

Sorgeplattformen sind natürlich kein Effekt der Coronakrise. Sie sind wie alle Plattformökonomien Ausdruck der Digitalisierung, der Finanzkrise und vor allem der Carekrise, der grundsätzlich fehlenden Anerkennung und des privaten Charakters von Sorgearbeit sowie des Abbaus von öffentlicher Versorgung. Anhand der vergeschlechtlichten und verräumlichten Strukturen der Sorgearbeit lässt sich einiges über das Wachstum digitaler Plattformen in der Stadt ableiten (Ecker/Rowek/Strüver 2021). Umgekehrt lässt sich auch skizzieren, was Sorgeplattformen hinsichtlich der Coronakrise und der Carekrise in der Stadt deutlich machen: Die Plattformisierung von Sorgearbeit basiert technologisch auf der Digitalisierung und sozioökonomisch auf der Flexibilisierung von Arbeitsverhältnissen. Sie findet aufgrund der Bevölkerungsdichte (von potenziellen Nutzer_innen wie von 
Arbeitskräften) vor allem in der Stadt statt und wird - auch jenseits von Sorgeplattformen - als Plattformurbanismus betitelt (Barns 2020).

Sorgeplattformen sind aber auch die ökonomisierte Antwort auf die Bewältigung von strukturell bedingten individuellen Zeit- und Sorgekrisen. Für Anbietende wie Nutzende bieten sie - in einem als informalisiert geltenden Sektor - eine formalisierte Vermittlung mit großem Angebot. Neben der Bevölkerungsdichte der Stadt spielt für ihren Erfolg die zeitliche Flexibilität, vor allem für Anbietende mit eigenen Sorgeverpflichtungen, eine wichtige Rolle und zugleich sind sie, wie alle Plattformökonomien, für ihre prekären Arbeitsbedingungen bekannt (Huws 2019).

Beide Plattformbeispiele machen deutlich, dass vergeschlechtlichte Sorgebeziehungen kein individuelles Problem sind, sondern ein strukturelles, das stark mit Klassen- und Migrationserfahrungen verbunden ist (Schwiter/ Steiner 2020): Urbane Plattformökonomien wie helpling.com vermarktlichen also Sorgearbeiten - es geht hier um die Bezahlung von reproduktiven Dienstleistungen für diejenigen, die es sich leisten können, und um die Verrichtung durch diejenigen, für die ein solches Einkommen unter anderem aufgrund der fehlenden Regulierung oder der hohen Flexibilität essenziell ist.

\section{Sorgende Urbanisierung}

Durch die Coronakrise zeichnen sich große gesellschaftliche Veränderungen ab - sie werden aber nur "the end of capitalism as we knew it" sein: Die Coronakrise bietet die Chance, „die aktuell stattfindenden Transformationen urbaner Ökonomien, Kulturen und Alltagspraktiken durch Digitalisierung und Pandemie durchaus ernst zu nehmen und ihre Wirkungen, Akteurskonstellationen und Ungleichheitsdynamiken zu beleuchten" (Höhne/ Michel 2021: 147). Ich möchte dies ausblickend im Sinne einer Transformation vom sorglosen Kapitalismus zur sorgenden Urbanisierung entlang von fünf Thesen und anhand der folgenden aus dem Aufschlag adaptierten Frage konkretisieren: Wie muss die kritische Stadtforschung die Transformation der urbanen Sorgearbeit im Krisenkapitalismus adressieren?

(1) Die Pandemie als Verdichtung der Körper im Wohnraum hat Funktionsmischung auf engstem Raum radikalisiert; dadurch hat die Coronakrise die Carekrise und die anhaltend untertheoretisierten Grundlagen kapitalistischer Gesellschaften, die Sorgearbeit, sicht- und spürbar gemacht. Wenn Corona und Care zusammen diskutiert werden, dürfen Kapitalismus und Krise nicht fehlen - und vice versa.

(2) Sorgearbeit als Teil der Alltagsökonomie muss als elementarer Bestandteil städtischer Ökonomien - analog wie digital - adressiert werden, gerade weil sie zu Hause und, privat' bzw. unsichtbar und unabgesichert stattfindet; Sorgearbeit ist nicht individuell, sondern strukturell.

(3) Plattformvermittelte Sorgearbeit hat das Modell unbezahlter Hausfrauenarbeit endgültig dekonstruiert, aber sie überkommt weder die Unsichtbarkeit der Sorgearbeit noch ihre Assoziationen mit Weiblichkeit und Privatheit. Zudem ist entlohnte Sorgearbeit äußerst prekarisiert und darf nicht als individuell flexible Lösung romantisiert werden: Sorgeplattformen als Segmente des Plattformurbanismus sind Teil des ungleichen Wirtschaftswachstums, in dem nur die Betreiber profitieren. 
(4) Sorgearbeiten müssen jenseits des Fokus auf den Gebrauchswert von Dienstleistungen als Grundlage aller wirtschaftlichen Aktivitäten akzeptiertwerden: Gibson-Graham(2006) haben mit ihrem Konzept der diverse economies gezeigt, dass profitorientiertes Wirtschaften nur einen kleinen Teil der alltäglich überlebenswichtigen ökonomischen Transaktionen ausmacht, die Spitze des Eisberges. Das weitaus größere Fundament des Eisberges ist hingegen unsichtbar. Ihr Konzept ist gleichwohl keine Kritik am kapitalistischen Wirtschaftssystem in dualistischer Manier, sondern eine Betonung des Gebrauchswerts (zum Beispiel Kochen) als Teil des Tauschwerts (zum Beispiel Lebensmittelerwerb). Dadurch verdeutlichen sie, dass Sorgearbeiten nicht nur die Reproduktion der Arbeitskräfte in entlohnten Tätigkeiten sichern, sondern Voraussetzung für kapitalistische Gesellschaftsbeziehungen sind.

(5) Coronakrise und Digitalisierung stellen die akute Rahmung der aktuellen Transformationen urbaner Ökonomien dar. Im Pandemischen Urbanismus wird das Fundament des Eisberges sicht- und spürbarer aber nur eine sorgende Urbanisierung als „minor theory“ (Katz 2017) im Verhältnis zur Planetarischen Urbanisierung macht auch die Carekrise theoretisch wie gesellschaftlich bearbeitbar.

Dieser Artikel wurde durch Mittel des Open Access Publikationsfonds der Karl-Franzens-Universität Graz gefördert.

\section{Endnoten}

[1] Als Carekrise wird der Zustand beschrieben, in dem die Nachfrage an bezahlter wie unbezahlter Sorgearbeit regional oder national höher ist als das Angebot. Der Begriff zielt also nicht auf Sorge- und Versorgungsdefizite in einzelnen Haushalten ab, sondern auf die gesellschaftliche Ebene.

[2] Reproduktionsarbeit steht hier für unbezahlte Tätigkeiten, die die Lohnarbeit im Kapitalismus ermöglichen. Als Sorgearbeit gelten hingegen die konkreten bezahlten und unbezahlten Tätigkeiten wie Kochen, Betreuen, Putzen, Pflegen etc. (Winker 2015: 17).

[3] Im Unterschied zur Produktivitätssteigerung bei Gütern oder Wissen usw. lassen sich z. B. Kinder und Kranke nicht, schneller betreuen und auch der Einsatz von Technologien hat sich bislang für körpernahe und beziehungsintensive Dienstleistungen als ungeeignet erwiesen.

[4] https://www.restaurantbusinessonline.com/operations/4-trends-defining-deliveryduring-covid-19

\section{Autor_innen}

Anke Strüver ist Humangeographin. Sie arbeitet mit einem feministischen Fokus zum urbanen Alltagsleben anhand von Verkörperungen, Gesundheit, Ernährung, Sorge/Versorgung und Digitalisierung.

anke.struever@uni-graz.at 


\section{Literatur}

Barns, Sarah (2020): Platform urbanism. Negotiating platform ecosystems in connected cities. Singapore: Palgrave.

Carstensen, Tanja (2020): Orts- und zeitflexibles Arbeiten: Alte Geschlechterungleichheiten und neue Muster der Arbeitsteilung durch Digitalisierung. In: Zeitschrift für Arbeitswissenschaft 74, 195-205.

Craig, Lyn (2020): Coronavirus, domestic labour and care. Gendered roles locked down. In: Journal of Sociology 56/4, 684-692.

Derndorfer, Judith / Disslbacher, Franziska / Lechinger, Vanessa / Mader, Katharina / Six, Eva (2020): Corona-Krise als Instrument der Gleichstellung? In: A\&W blog, 30.6.2020. https://awblog.at/corona-krise-als-instrument-der-gleichstellung/ (letzter Zugriff am 18.1.2021).

van Doorn, Niels / Mos, Eva / Bosma, Jelke (2020): Disrupting „business as usual“. How COVID-19 is impacting platform-mediated labor and social reproduction. https:// platformlabor.net/blog/disrupting-business-as-usual (letzter Zugriff am 18.1.2021).

Ecker, Yannick / Rowek, Marcella / Strüver, Anke (2021): Care on Demand: Geschlechternormierte Arbeits- und Raumstrukturen in der plattformbasierten Sorgearbeit. In: Moritz Altenried / Julia Dück / Mira Wallis (Hg.), Plattformkapitalismus und die Krise der sozialen Reproduktion. Münster: Westfälisches Dampfboot.

Federici, Silvia (2019): Re-enchanting the world. Feminism and the politics of the commons. Oakland: PM Press.

Gibson-Graham, J. K. (2006): The end of capitalism (as we knew it). A feminist critique of political economy. Minneapolis: Minnesota UP (2. Auflage).

Höhne, Stefan / Michel, Boris (2021): Das Ende des Städtischen? Pandemie, Digitalisierung und planetarische Enturbanisierung. In: sub\urban. zeitschrift für kritische stadtforschung 9/1-2, 141-149.

Huws, Ursula (2019): The hassle of housework. Digitalization and the commodification of domestic labour. In: Feminist Review 123/1, 8-23.

Katz, Cindi (2017): Revisiting minor theory. In: Environment and Planning D: Society and Space 35/4, 596-599.

Kohlrausch, Bettina / Zucco, Aline (2020): Die Corona-Krise trifft Frauen doppelt. In: Policy Brief WSI 40, 1-14.

Schuster, Nina / Höhne, Stefan (2017): Stadt der Reproduktion. Einführung in den Themenschwerpunkt. In: sub\urban. zeitschrift für kritische stadtforschung 5/3, 9-22.

Schwiter, Karin / Steiner, Jennifer (2020): Geographies of care work. The commodifiation of care, digital care futures and alternative caring visions. In: Geography Compass 14/12, 1-16.

Soiland, Tove (2020): New modes of enclosures. A feminist perspective on the transformation of the social. In: Fabian Kessl / Walter Lorenz / Hans-Uwe Otto / Sue White (Hg.), European social work. A compendium. Opladen: Budrich, 289-318.

Winker, Gabriele (2015): Care Revolution. Schritte in eine solidarische Gesellschaft. Bielefeld: transcript. 